\title{
Preparation of Graphene Quantum Dots and Their Application in Cell Imaging
}

\author{
Jie Zhang, ${ }^{1}$ Yong-qiang Ma, ${ }^{2} \mathrm{Na} \mathrm{Li}^{3}{ }^{3}$ Jing-li Zhu, ${ }^{3}$ Ting Zhang, ${ }^{3}$ Wei Zhang, ${ }^{3}$ and Bin Liu \\ ${ }^{1}$ School of Nuclear Science and Technology and School of Stomatology, Lanzhou University, Lanzhou 730000, China \\ ${ }^{2}$ Department of Stomatology, The Second People's Hospital of Gansu Province, Lanzhou 730000, China \\ ${ }^{3}$ School of Stomatology, Lanzhou University, Lanzhou 730000, China \\ Correspondence should be addressed to Bin Liu; liubkq@lzu.edu.cn
}

Received 21 January 2016; Accepted 20 April 2016

Academic Editor: Miguel A. Correa-Duarte

Copyright (C) 2016 Jie Zhang et al. This is an open access article distributed under the Creative Commons Attribution License, which permits unrestricted use, distribution, and reproduction in any medium, provided the original work is properly cited.

\begin{abstract}
Objective. This study aims to increase the fluorescence quantum yield by improving the conditions of preparing graphene quantum dots (GQDs) through the solvothermal route and observe the GQDs performance in imaging oral squamous cells. Methodology. The following experimental conditions of GQDs preparation through the solvothermal route were improved: graphene oxide (GO)/N$\mathrm{N}$ dimethyl formamide (DMF) ratio, filling percentage, and reaction time. A fluorescence spectrophotometer was used to measure photoluminescence, and the peak values were compared. Methylthiazolyldiphenyl-tetrazolium (MTT) bromide was used to detect the cytotoxicity of GQDs, which was compared with that of cadmium telluride quantum dots (CdTe QDs). GQDs were cultured with tongue cancer cells. After the coculture, a laser scanning confocal microscope (LSCM) was used to observe cell imaging. Results. The optimal conditions of GQD preparation through the solvothermal route included the following: $10 \mathrm{mg} / \mathrm{mL} \mathrm{GO} / \mathrm{DMF}$ ratio, $80 \%$ filling percentage, $12 \mathrm{~h}$ reaction time, and $17.4 \%$ fluorescence quantum yield. As the cell concentration increased, the GQD and CdTe QD groups exhibited a decreasing cell survival rate, with the decrease in the CdTe QD group being more significant. The LSCM observations showed bright green fluorescence images. Conclusion. The improved experimental conditions increased the fluorescence quantum yield of GQDs. In this study, the prepared GQDs exhibited low cytotoxicity level and satisfactory cell imaging performance.
\end{abstract}

\section{Introduction}

Graphene quantum dots (GQDs) are zero-dimensional graphene nanoparticles with excellent optical properties and biosecurity. For these traits, the application of GQDs shows remarkable prospects in biomedical fields, such as cell imaging $[1,2]$ and biosensors [3]. Currently, various techniques have been developed to produce GQDs. Bottom-up methods include solution chemical [4], microwave [5], and ultrasonic methods [6]. Up-bottom methods include hydrothermal [7] and electrochemical methods [8]. These techniques are complex and have lower fluorescence quantum yield and intensity compared to conventional semiconductor quantum dots (QDs) and carbon QDs. Zhu et al. [9] applied the solvothermal route to produce GQDs and achieved a QD yield of $11.4 \%$. In the present study, the solvothermal route was improved to increase fluorescence quantum yield of
GQDs by $17.4 \%$. The produced GQDs have good stability and lower cytotoxicity compared to CdTe QDs. In addition, the produced GQDs can easily enter cells. Our finding was intended to provide a research direction for the application of GQDs in cell imaging and protein labeling.

\section{Materials and Methods}

2.1. Main Reagents and Equipment. The following reagents and equipment were used in this study: CdTe QDs (Nanjing Janus New Materials Co., Ltd., China), dialysis bag (EI9700, MD-25, NMCO: 3500, Beijing Huamei Scientific Co.), digital temperature magnetic stirrer (Jinyan, 85-2), Muffle Furnace (MF-1100C-S, Anhui BEQ Equipment Technology Co., Ltd.), Transmission Electron Microscope (JEOL 2011, Philips-CM20, Holland), Fluorescence Spectrometer (Cary Eclipse, Varian), UV-vis spectrophotometer (TU-1901, 
Beijing Purkinje General Instrument Co., Ltd.), microplate reader (PerkinElmer, Waltham, MA, USA), laser scanning confocal microscope (Olympus, USA), and Fluorescence Spectrometer (Cary Eclipse, Japan).

2.2. GQD Preparation Using the Solvothermal Route. Graphene oxide (GO) was prepared through improved Hummers method, and GQDs were prepared through the solvothermal route. The preparation steps were as follows: $480 \mathrm{mg}$ of GOs was added in $48 \mathrm{~mL}$ of N-N dimethyl formamide (DMF) to produce $10 \mathrm{mg} / \mathrm{mL}$ GO/DMF suspension. The suspension was treated with ultrasonic dispersion $(500 \mathrm{~W})$ for $1 \mathrm{~h}$, transferred into a $60 \mathrm{~mL}$ Teflon bottle held in a stainless steel autoclave, and heated in the muffle furnace to $200^{\circ} \mathrm{C}$ for $8 \mathrm{~h}$. The final GQD/DMF products can be obtained through vacuum filtration using a $0.22 \mu \mathrm{m}$ micropore filter membrane. The GQD/DMF suspension was rotated for evaporation to remove DMF and obtain GQDs, which were then dissolved in different solvents, such as pure water, normal saline, and phosphate buffered saline (PBS) to produce different suspensions.

The GO/DMF ratios of $0.5,1,5,10$, and $20 \mathrm{mg} / \mathrm{mL}$ were strictly controlled. The filling degrees in the reaction were $40 \%, 60 \%$, and $80 \%$. The reaction times were 4,8 , and $12 \mathrm{~h}$. The GQD/DMF samples were individually produced through the above-mentioned experimental methods. To select the optimal preparation conditions, the fluorescence quantum yields of the different GOD samples were measured using fluorescence spectroscopy (excitation wavelength, Ex: $420 \mathrm{~nm}$, with quinine sulfate as the reference). The calculated formula was as follows:

$$
\text { Фst can be described as }\left(\frac{I x}{I s t}\right)\left(\frac{\eta^{2} x}{\eta^{2} s t}\right)\left(\frac{A s t}{A x}\right) \text {, }
$$

where $\Phi$ is the fluorescence quantum yield, $I$ is the emission intensity, $\eta$ is the refractive index of solvents, $A$ is the UV absorption at $425 \mathrm{~nm}, x$ is the GQDs, and st is the standard controls.

2.3. GQDs Cytotoxicity. Human tongue cancer Tca 8113 cells were cultured in RPMI1640 medium supplemented with 10\% fetal calf serum, which was placed in the incubator at $37^{\circ} \mathrm{C}$ with $5 \% \mathrm{CO}_{2}$ and saturated humidity. The nutrient solution was replaced every $2 \mathrm{~d}$. In $2 \mathrm{~d}$ to $3 \mathrm{~d}, 0.25 \%$ trypsin was used to digest the cells and enable passage. The solution was transformed into a single-cell suspension and inoculated in a 96-pore plate with $1 \times 10^{4}$ cells/pore. The total amount of medium was $200 \mu \mathrm{L}$ per pore. GQDs were added to the 96pore plate of each group. The final concentration in each group was regulated at $0,100,200,300$, and $400 \mu \mathrm{g} / \mathrm{mL}$, and cells were cultured in an incubator at $37^{\circ} \mathrm{C}$ with $5 \%$ $\mathrm{CO}_{2}$ for $24 \mathrm{~h}$. MTT liquid ( $5 \mathrm{mg} / \mathrm{mL}$ and $20 \mu \mathrm{L} /$ pore) was then added to the 96-pore plate, and the culture time was extended for another $4 \mathrm{~h}$. The nutrient solution in the pores was extracted and then replaced by dimethyl sulfoxide liquid $(150 \mu \mathrm{L} /$ pore). The plate was placed in a microplate oscillator for $10 \mathrm{~min}$ of oscillation at room temperature. The crystals were dissolved through this approach, and the microplate reader was analyzed under the wavelength of $490 \mathrm{~nm}$ to detect OD in each pore. The results were then recorded. An equivalent amount of CdTe QDs was added to the control group.

Calculation of the survival rate of cells: the survival rate of cells (cell viability) was calculated according to the following equation:

$$
\text { cell viability }(\%)=\left(\frac{\mathrm{OD}_{\text {treated }}}{\mathrm{OD}_{\text {control }}}\right) \times 100 \% \text {, }
$$

where $\mathrm{OD}_{\text {treated }}$ denotes the addition of GQDs or CdTe QDs and $\mathrm{OD}_{\text {control }}$ means no addition of GQDs or CdTe QDs. To ensure reliability of the experimental data, each sample was repeatedly analyzed five times, and the average value and standard deviation were generated to draw images.

Observation of cell morphology: the 96-pore plate was placed under an optical microscope to observe cell morphology. Photos were subsequently taken.

\subsection{Cell Imaging of GQDs}

2.4.1. Single-Cell Staining. After the digestion of Tca8113 cells at the logarithmic growth phase with $0.25 \%$ trypsin, the cells were inoculated with density of $1 \times 10^{6}$ cells $/ \mathrm{mL}$ in the 6pore plate with a sterile cover slip. The plate was placed in an incubator at $37^{\circ} \mathrm{C}$ with $5 \% \mathrm{CO}_{2}$ and saturated humidity for $24 \mathrm{~h}$. After cell adhesion, the nutrient solution was removed, and the cells were rinsed with PBS three times. Afterward, $4 \%$ paraformaldehyde was used to fix the cells for $30 \mathrm{~min}$. The cells were rinsed with PBS two times to remove excess paraformaldehyde. Briefly, $500 \mu \mathrm{L}$ of $0.4 \mathrm{mg} / \mathrm{mL}$ GQD was added in $500 \mu \mathrm{L}$ of RPMI1640 medium and cocultured with fixed cells for $24 \mathrm{~h}$. The slides were sealed with propolis and observed under LSCM (Ex: 405 nm, Em: 535 nm).

2.4.2. Double Staining of the Cytoplasm and Nucleus. Tca8113 oral squamous cells at the logarithmic phase were digested with $0.25 \%$ trypsin and inoculated with density of $1 \times$ $10^{6}$ cells $/ \mathrm{mL}$ in a 6 -pore plate with a sterile cover slip. The cells were cultured in the incubator at $37^{\circ} \mathrm{C}$ with $5 \%$ $\mathrm{CO}_{2}$ under saturated humidity for $24 \mathrm{~h}$. After cell adhesion, the cells were rinsed thrice with PBS and then fixed with $4 \%$ paraformaldehyde for $30 \mathrm{~min}$. To remove excess paraformaldehyde, the fixed cells were rinsed twice with PBS. Subsequently, $500 \mu \mathrm{L}$ of $(0.4 \mathrm{mg} / \mathrm{mL})$ GQD was added in $500 \mu \mathrm{L}$ of RPMI1640 medium and cocultured with fixed cells for $24 \mathrm{~h}$. After removing the cellular culture solution, a solution of Triton X-100 was added, and the cells were cultured at room temperature for $10 \mathrm{~min}$. The cultured cells were rinsed thrice with PBS. The inorganic fluorochrome Hoechst 33258 stain was added and cocultured with cells for $20 \mathrm{~min}$. To remove excess stain, the cultured cells were rinsed thrice with PBS. Afterward, the slides were sealed with propolis. An equivalent amount of pure water was added for the control group. Samples were observed under LSCM (Ex: $405 \mathrm{~nm}$ ) to measure the luminance of GQD and then under LSCM (Ex: $488 \mathrm{~nm}$ ) to identify and determine luminance 

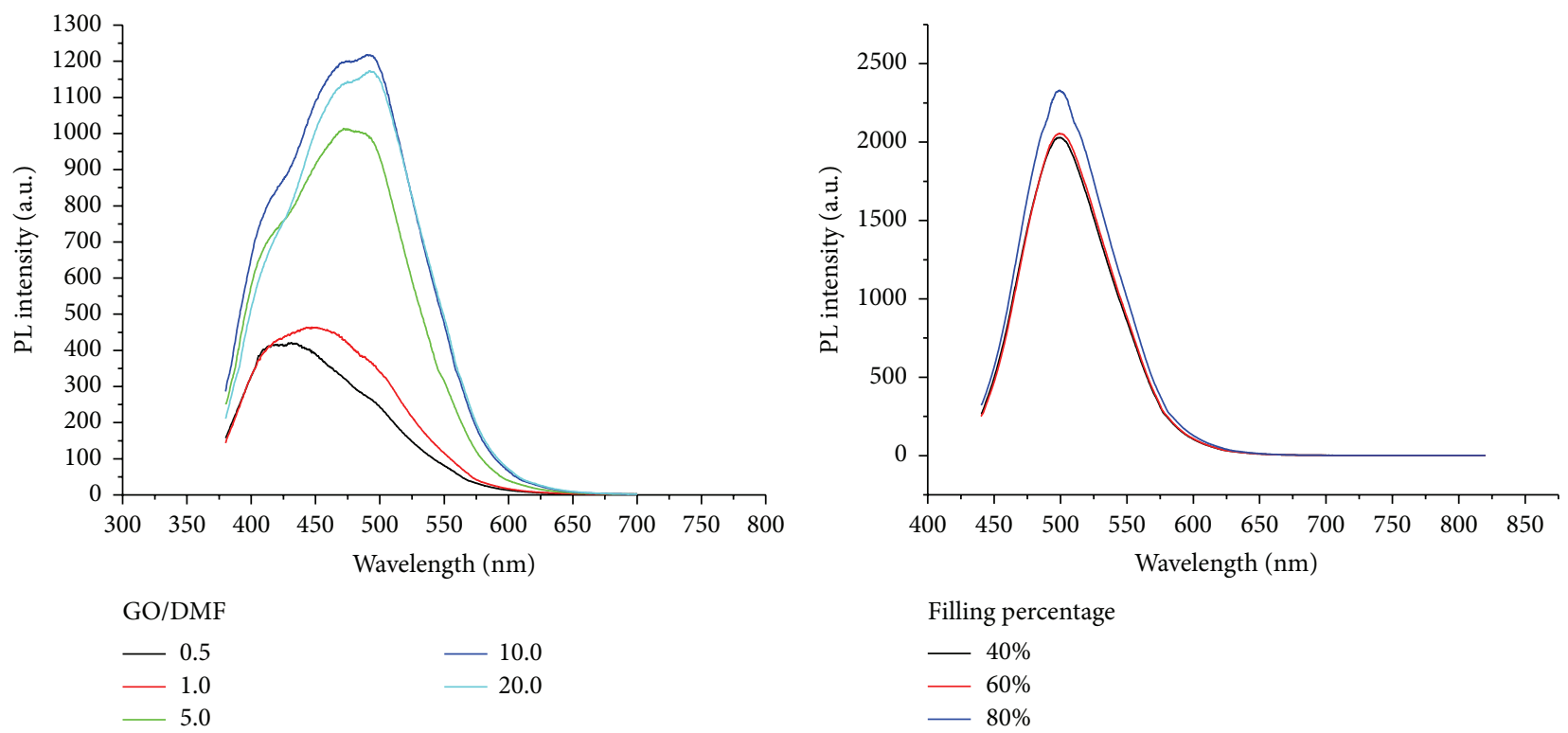

(a)

(b)

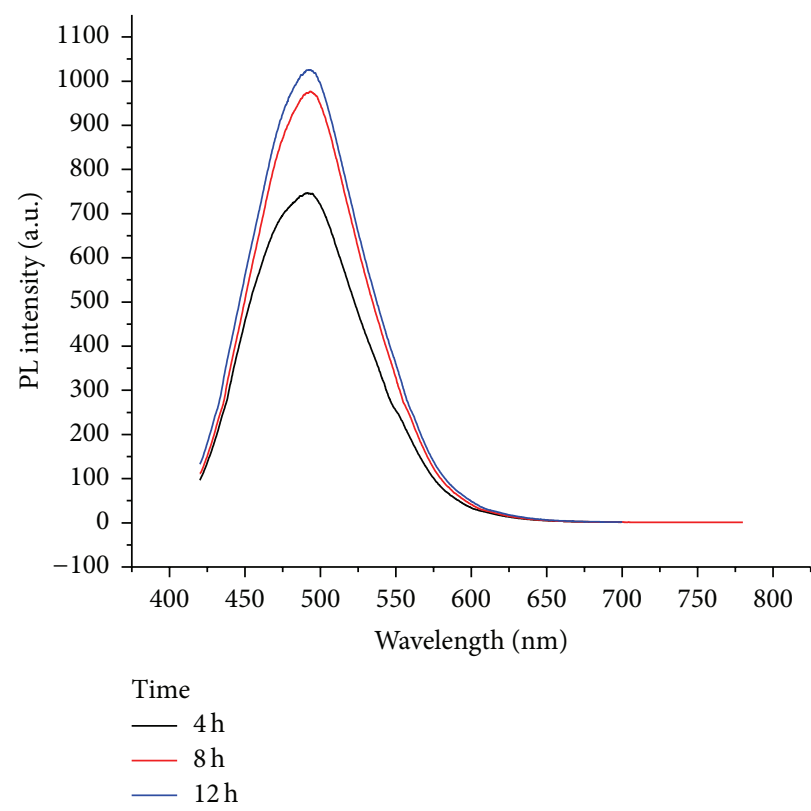

(c)

FIGURE 1: PL spectra under different experimental conditions of preparation of GQDs. (a) Different GO/DMF ratio; (b) different degrees of filling; and (c) different reaction time (Ex: $420 \mathrm{~nm})$.

of the cell nucleus. Subsequently, the obtained images were overlapped.

\section{Results}

3.1. Optimal Preparation Conditions for GQDs with the Solvothermal Route. By controlling the different synthesis conditions (GO/DMF ratios, filling percentages, and reaction time), different GQDs were prepared and assayed through photoluminescence (PL) spectroscopy. According to the findings, the optimal preparation conditions for GQDs through the solvothermal route include $10 \mathrm{mg} / \mathrm{mL} \mathrm{GO} / \mathrm{DMF}$ ratio, $80 \%$ filling percentage in the reaction, $200^{\circ} \mathrm{C}$ temperature in the muffle furnace, and $12 \mathrm{~h}$ reaction time (Figure 1 ). Using the measurement method in the literature [9], the fluorescence quantum yield of GQDs produced under the optimal conditions was $17.4 \%$ (Table 1), which was higher compared to 
TABLE 1: Quantum yield of GQDs using quinine sulfate as a reference.

\begin{tabular}{lcccc}
\hline Sample & Integrated emission intensity $(I)$ & Abs. at $425 \mathrm{~nm}(A)$ & Refractive index of solvent $(\eta)$ & Quantum yield $(\Phi)$ \\
\hline Quinine sulfate & 340312.957 & 0.020 & 1.33 & 0.54 \\
GQDs & 252362.705 & 0.046 & 1.33 & 0.174 \\
\hline
\end{tabular}

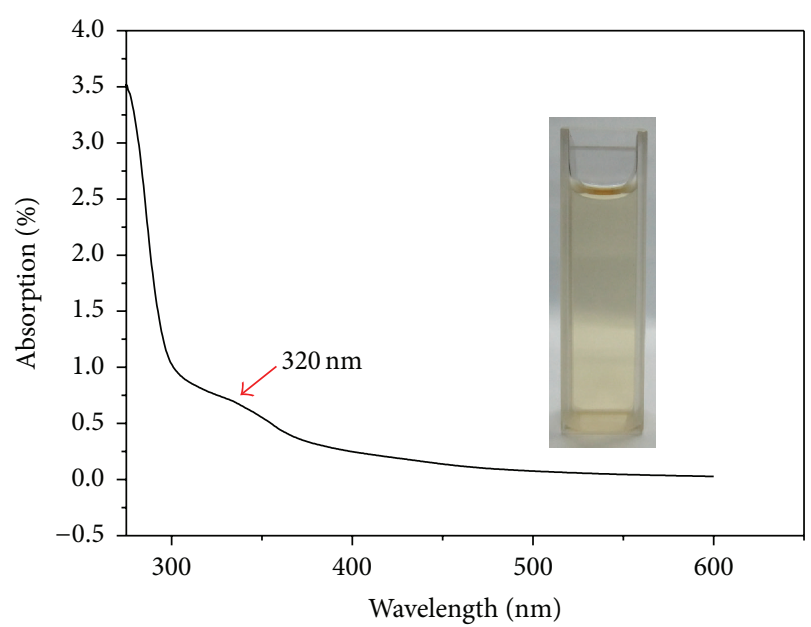

(a)

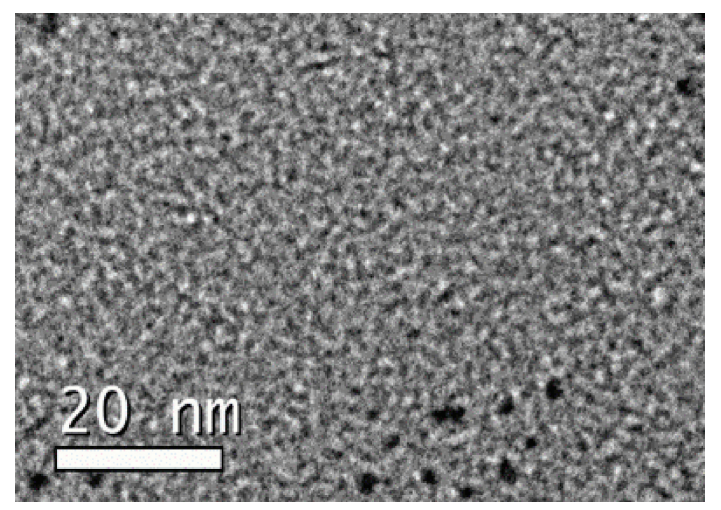

(c)

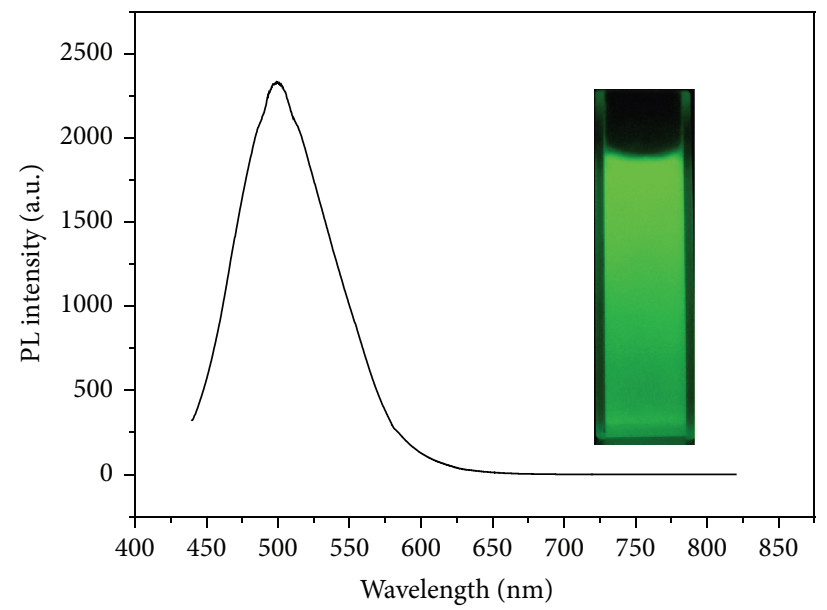

(b)

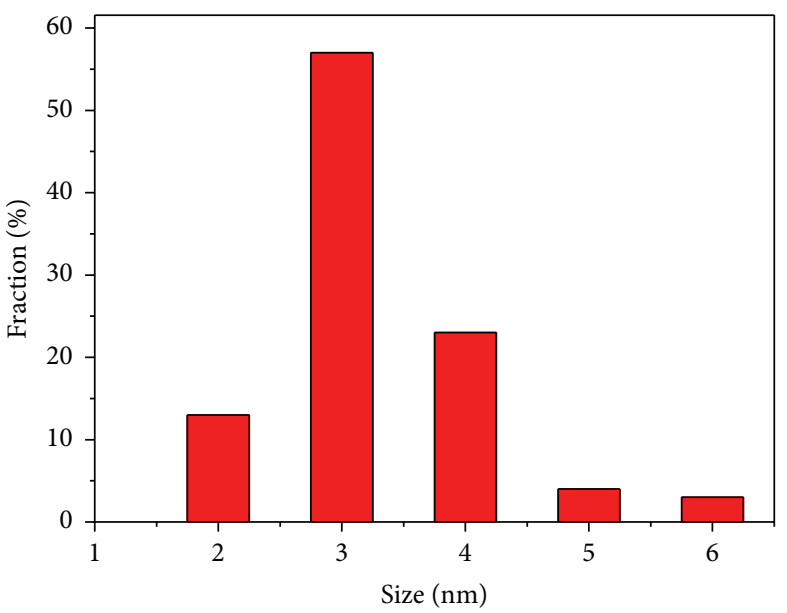

(d)

FIGURE 2: Characterization of GQDs. (a) UV-vis spectra of GQDs; (b) PL spectra of GQDs (Ex: $420 \mathrm{~nm}$ ); (c) TEM image of the GQDs (average size $3.783 \mathrm{~nm}$ ); and (d) size distributions of GQDs.

the fluorescence quantum yield of GQDs produced through the method of Zhu et al. [9].

\subsection{Representation of Graphene Quantum Dots. Figure 2(a)} shows the UV-vis spectra of the GQD aqueous solution produced under optimal conditions. An evident absorption peak was observed at a wavelength of $320 \mathrm{~nm}$, which is consistent with that in literature [8]. In the PL spectrum (Figure 2(b)), at the excitation wavelength of $420 \mathrm{~nm}$, the highest peak appeared at $500 \mathrm{~nm}$, and the full width at half maximum was approximately $50 \mathrm{~nm}$. This result was similar to that of the semiconductor and carbon QDs reported in the literature $[10,11]$. The images in the right corner of Figures 2(a) and 2(b) are digital images of the GQD pure water suspension under natural light and $365 \mathrm{~nm}$ UV excitation. The figures show the sufficient dispersion stability of GQDs in pure water, thus showing a faintly yellow transparent liquid. However, this sample illuminated strong green fluorescence under UV excitation. Figures 2(c) and 2(d) showed the TEM images and particle size distribution images of the GQDs, respectively. The average particle size was $3.783 \pm 0.829 \mathrm{~nm}$.

The GQD/DMF suspension exhibited different PL intensities at different Ex wavelengths, as shown in Figure 3(a). In Ex of $360 \mathrm{~nm}$ to $420 \mathrm{~nm}$, the PL intensity increased with 


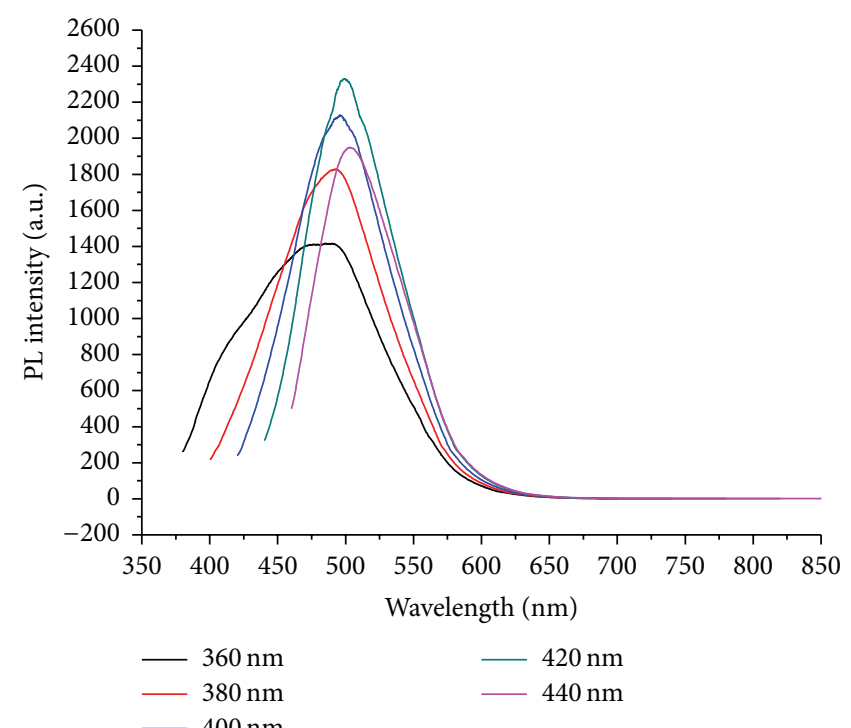

(a)
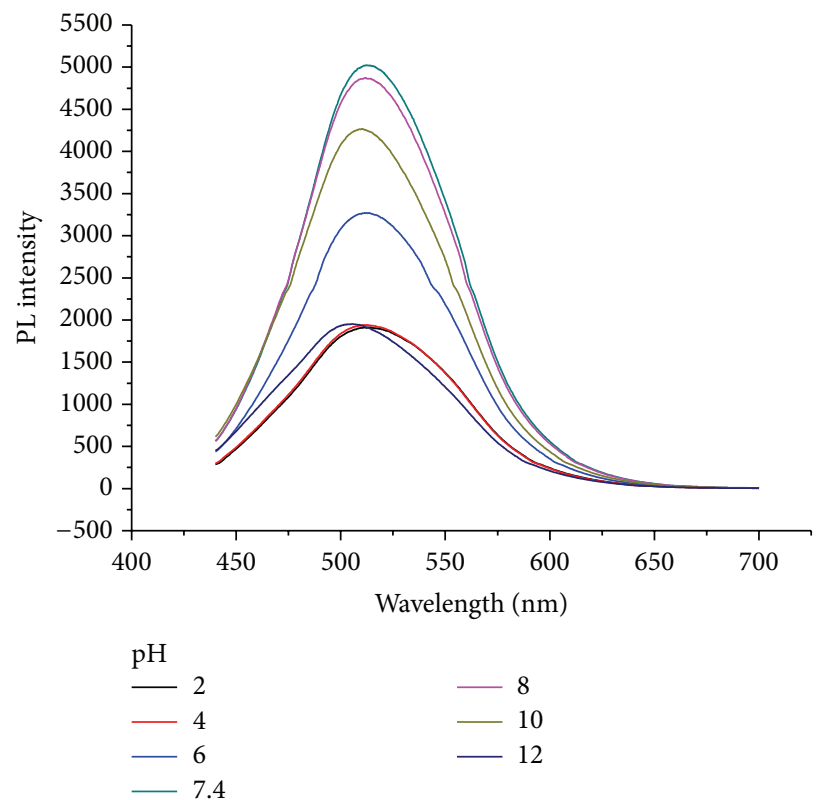

(c)

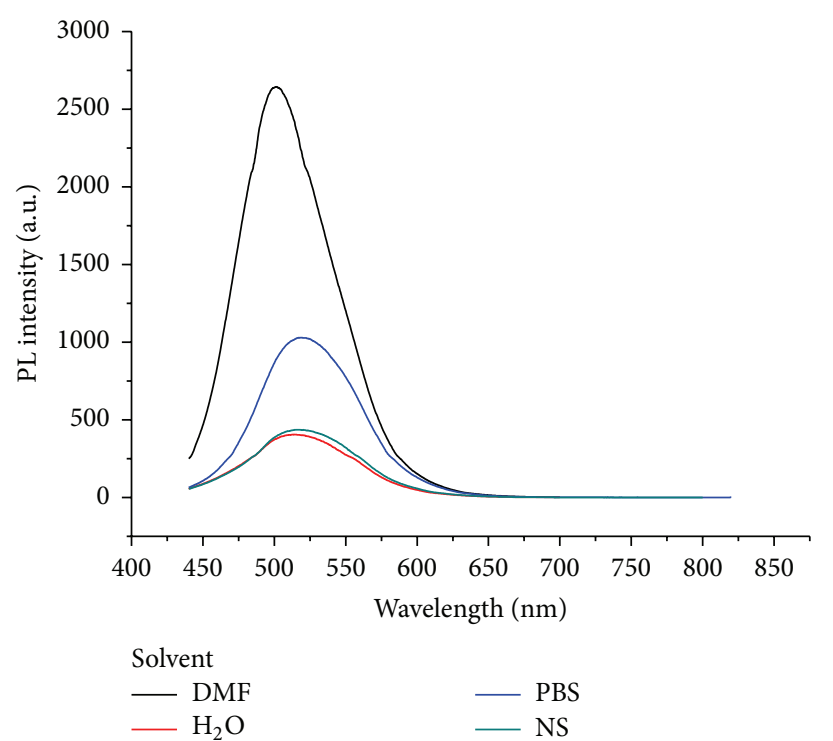

(b)

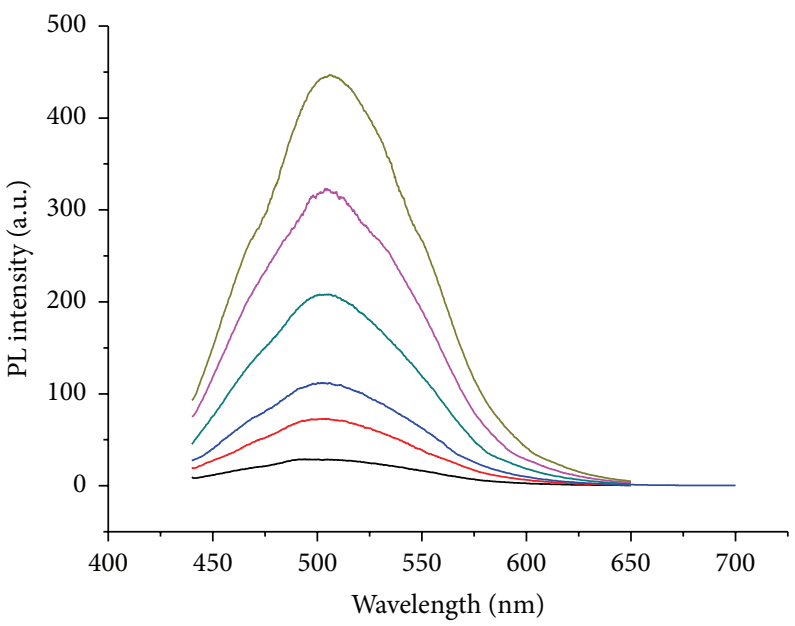

$\begin{array}{ll}\text { Concentration }(\mathrm{mg} / \mathrm{mL}) & \\ -0.025 & -0.200 \\ -0.050 & -0.500 \\ -0.100 & -1.000\end{array}$

(d)

FIGURE 3: The PL spectrum of GQDs under different conditions. (a) Different wavelengths Ex; (b) different solvent; (c) different PH; and (d) different concentrations (b, c, d, Ex: $420 \mathrm{~nm}$ ).

increasing Ex wavelengths and PL peak shifted from $475 \mathrm{~nm}$ to $500 \mathrm{~nm}$. When the Ex wavelength exceeded $420 \mathrm{~nm}$, the PL intensity decreased. Figure 3(b) demonstrates that the PL intensities of GQD also differed in various solvents. In DMF, the PL intensity was significantly higher than that in other inorganic solvents. The maximum PL intensity of the GQD/PBS suspension was observed in a neutral environment. Excess acids or alkalis would reduce the intensity (Figure $3(\mathrm{c})$ ), and the changes in the intensities are faster in acidic environments than in basic environments (Figure 4(a)). The PL intensity also increased with increasing GQD/NS suspension concentration (Figure 4(b)).
3.3. Cytotoxicity of Graphene QDs. The MTT results showed that the survival rate of the cells in the GQD group was higher than that of the CdTe QD group at low concentration. When the concentration reached $300 \mu \mathrm{g} / \mathrm{mL}$, the survival rate of the cells in the GQDs group remained higher than $80 \%$, whereas that of the CdTe QDs group was lower than $60 \%$. However, with the concentration increasing to $400 \mu \mathrm{g} / \mathrm{m}$, the survival rate of cells in GQDs group decreased to $48 \%$, with no significant differences with CdTe QDs group (49\%) (Figure 5). LSCM observations demonstrated (Figure 6) that when the concentration reached $300 \mu \mathrm{g} / \mathrm{mL}$, the cellular morphology in the GQD group remained normal. Cell showed 


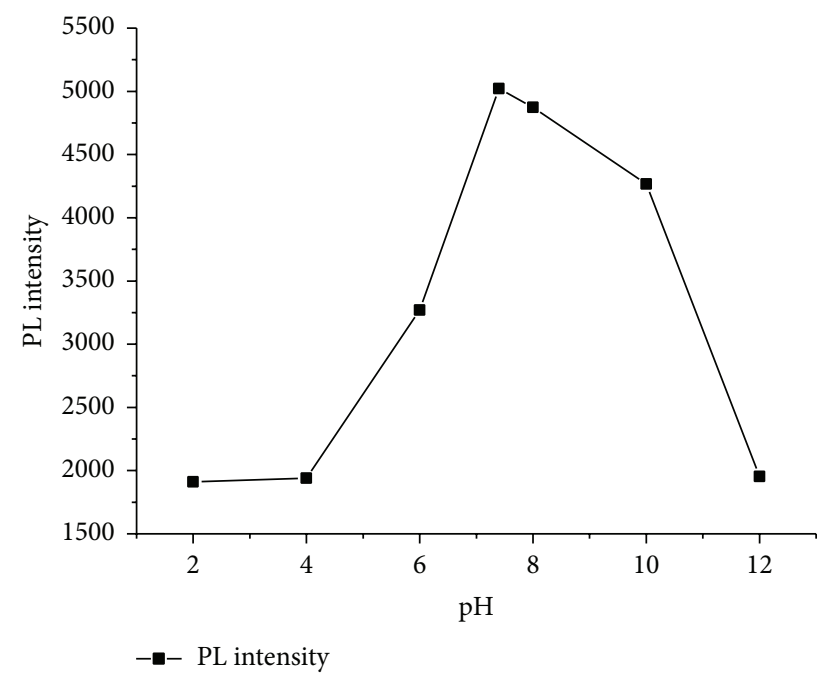

(a)

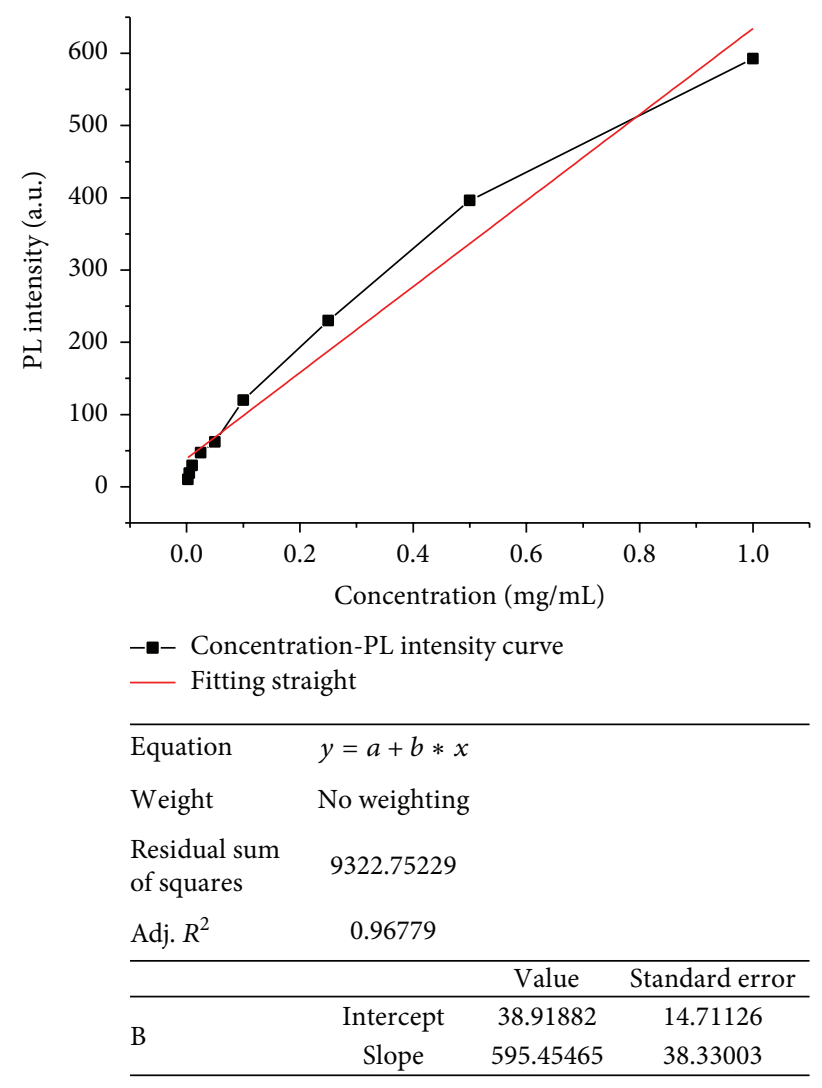

(b)

Figure 4: The peak value of PL spectrum under different $\mathrm{PH}$ and concentrations. (a) Different PH. (b) Different concentrations.

a cobblestone-like appearance and cellular tuber was distinct. However, most cell processes disappeared and became round or oval in the CdTe QD group, indicating that most cells died. According to the results of the MTT experiment and microscopic observation, the cytotoxicity of GQDs was lower than that of the CdTe QDs, and the cytocompatibility of GQDs was better.

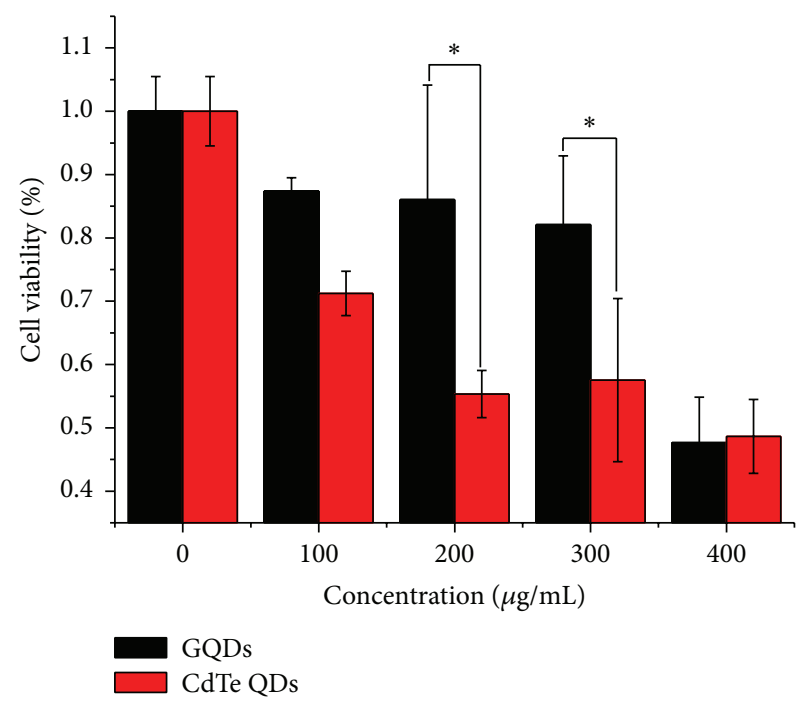

Figure 5: The cytotoxicity of GQDs ${ }^{*}(p<0.05)$.

3.4. Cell Imaging of Graphene QDs. When GQDs and Tca8113 cells were cocultured for $24 \mathrm{~h}$, results of the LSCM observations showed that, under $405 \mathrm{~nm}$ UV excitation, the cells showed bright green images (Figure 7). This finding indicates that GQDs can enter cells and are mainly concentrated in the cytoplasm without entering the cell nucleus. In addition, the fluorescence signals were significantly stronger in several areas of the cytoplasm, which may be attributed to the uneven aggregate distribution of GQDs in the cytoplasm. When the concentration of GQDs was high in several areas, the fluorescence signals were strong. This finding verified that GQDs could sufficiently form in vitro images of oral squamous cell Tca8113.

To better observe the imaging effects, double staining of the cytoplasm and nucleus was performed. Considering that GQDs could not enter the cell nucleus, the organic fluorochrome Hoechst 33258 stain was selected to dye the cell nucleus. The final obtained images were overlapped using different excitation wavelengths. Figure 8 shows that the green fluorescence of GQDs mainly concentrated in the cytoplasm, and the blue Hoechst 33258 stain fluorescence focused mainly around the area of the cell nucleus. The blue fluorescence disappeared after $1 \mathrm{~min}$ of continuous excitation, indicating that the organic fluorochrome was rapidly quenched. However, the green fluorochrome intensity of GQDs remained unchanged, indicating that their fluorochrome stability was higher than that of the organic fluorochrome.

\section{Discussion}

Numerous methods are available to produce graphene QDs, including ultrasonic, microwave, solution chemistry, hydrothermal, and electrochemical methods, as well as the solvothermal route. However, GQDs produced through these methods exhibit a significantly lower fluorescence quantum yield compared to semiconductor and carbon QDs [12-14]. The fluorescence quantum yield of the latter can reach at 


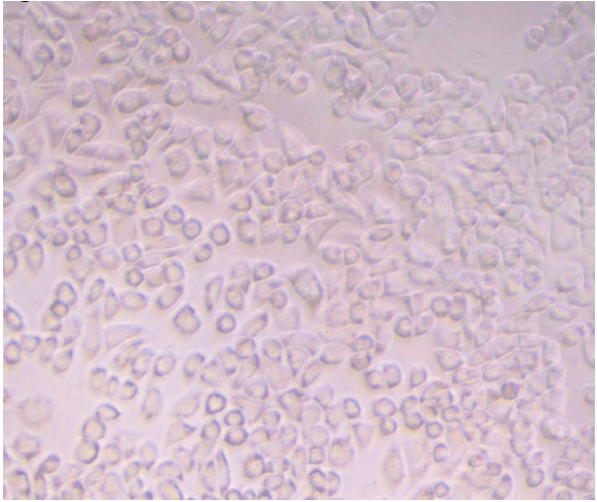

(a)

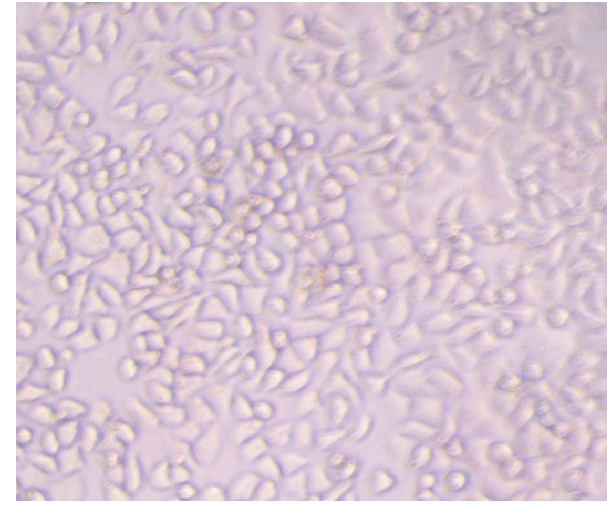

(b)

FIGURE 6: Cell morphological observated at $300 \mu \mathrm{g} / \mathrm{mL}$. (a) CdTe QDs group: most cell ecptomas vanished gradually and cell bodies became smaller and smoother; (b) GQDs group: it showed a cobblestone-like appearance and cellular tuber was distinct.

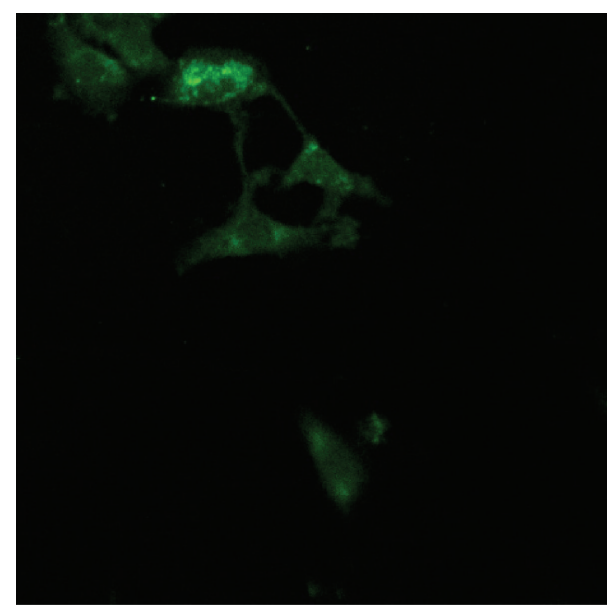

FIgURE 7: Under $405 \mathrm{~nm}$ UV excitation. The cells showed bright green images in the cytoplasm $(\times 400)$.

least $50 \%$. In our study, the solvothermal route was selected for the preparation of GQDs because this method is simple, easy to control, and safe. By controlling the different preparation conditions, the present study determined the following optimal preparation conditions: $10 \mathrm{mg} / \mathrm{mL}$ GO/DMF ratio, $80 \%$ filling percentage in the reaction, and $12 \mathrm{~h}$ reaction in the muffle furnace at $200 \mathrm{t}$. In this study, the fluorescence quantum yield of GQDs reached $17.4 \%$, which is higher than that reported by Zhu et al. During the reaction, DMF acted as the solvent as well as a weak reduction agent. The big GO was split and reduced simultaneously, and thus the GQDs were obtained.

The PL of the GQDs shows dependence on the excitation wavelength and $\mathrm{pH}$ value of the solution. Thus, the PL intensities at different excitation wavelengths and $\mathrm{pH}$ values in solutions are different. However, the peak position of PL remained unchanged. This phenomenon may be attributed to the different luminous mechanisms of GQDs compared with that of semiconductor [15] and carbon QDs [16]. The luminescence might be caused by the particle size or surface defects [1,17]. Meanwhile, the emission intensity of the GQDs in strong acidic and alkaline conditions decreased considerably because the $\mathrm{pH}$ change can induce protonation or deprotonation of emissive sites (i.e., oxygen groups and free zigzag sites) in the GQD cores $[18,19]$. However, around the optimum $\mathrm{pH}$ value of 7 for biosome, greater fluorescence of GQDs was detected, which was better than $\mathrm{CdSe} / \mathrm{ZnSe} / \mathrm{ZnS}$ quantum dots with stronger fluorescence in aqueous alkaline medium and extremely sensitive to $\mathrm{pH}$ [20]. With high PL quantum yield and greater fluorescence at $\mathrm{pH}$ of 7 , GQDs were appropriate for bioimaging. In addition, with increasing GQD concentration, the intensity of PL increased. Within the concentration range of $0 \mathrm{mg} / \mathrm{mL}$ to $1 \mathrm{mg} / \mathrm{mL}$, the PL intensity was linearly correlated with the concentration. Thus, in future studies, the PL distribution and metabolism in cells and human body can be detected by measuring the intensity of $\mathrm{PL}$ in tissue fluids, blood, or tissue homogenate.

Semiconductor QDs have been used in the biomedical research fields at an early stage because of their excellent optical properties. Compared with conventional organic fluorophores, the semiconductor QDs have a narrow, tunable, symmetric emission spectrum and photochemical stability $[10,21]$. However, the toxicity of semiconductor QDs in cells and human body is limiting their extensive application in biomedical fields [15-17]. Therefore, in recent years, related studies have focused on carbon materials with good biocompatibility. This study also proved that the cytotoxicity of GQDs is lower than that of CdTe QDs especially in lower concentration. In addition, GQDs have unique optical properties. In theoretical and experimental studies, quantum confinement and edge effects of "small" graphene (less than $10 \mathrm{~nm}$ ) have been shown to induce photoluminescence (PL) $[22,23]$. Due to quantum confinement effect, the functionalities of quantum dots can be simply tailored by controlling the size. Consequently, quantum dots have photochemical stability. In this experiment, GQDs can easily enter cells and are gathered in the cytoplasm. The imaging stability of GQDs was significantly better than that of organic fluorochrome, suggesting the sound prospects of using GQDs in the biological imaging field. Although GQDs have showed stronger 


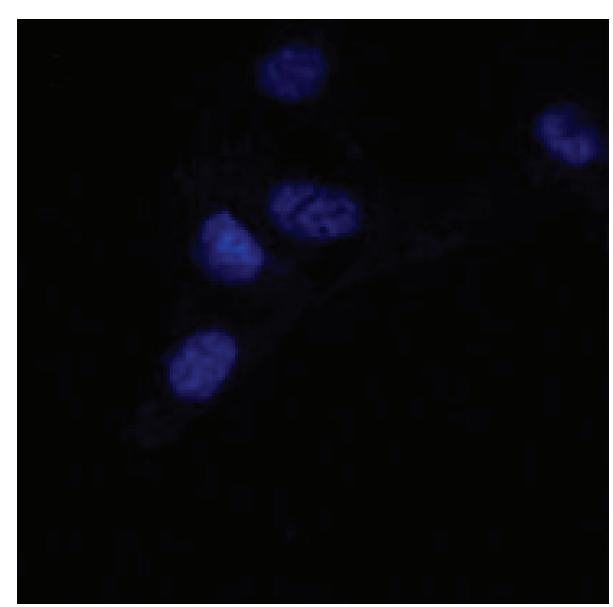

(a)

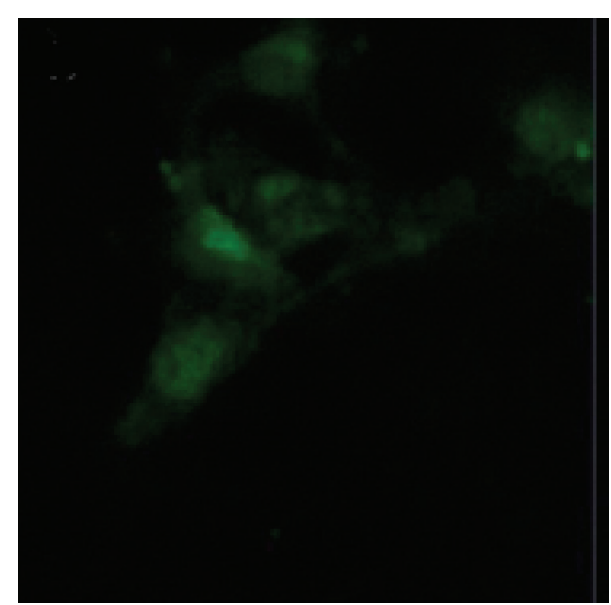

(b)

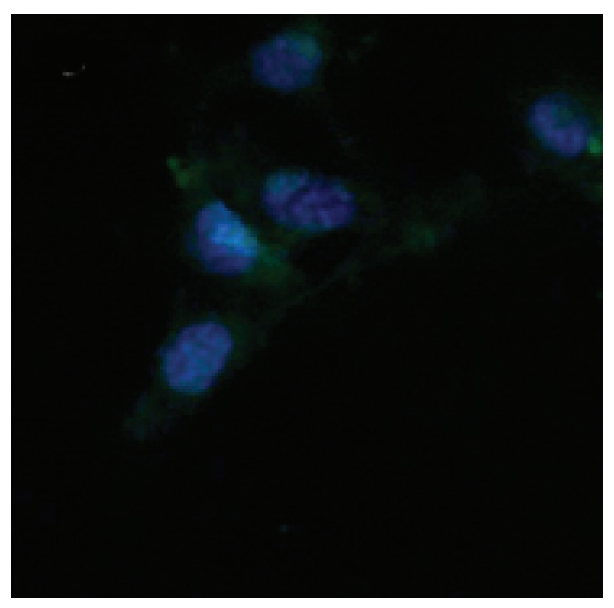

(c)

FIGURE 8: Double staining of the cytoplasm and nucleus. (a) Hoechst 33258 stain fluorescence focused mainly around the area of the cell nucleus (Ex: $488 \mathrm{~nm}$ ); (b) GQDs mainly concentrated in the cytoplasm (Ex: $405 \mathrm{~nm}$ ) (c) additive image of (a) and (b) $(\times 400)$.

stability than that of organic fluorochrome, PL intensity has also been influenced by the size of GQDs, $\mathrm{pH}$ value, solution, and excitation wavelength. The photochemical stability of GQDs should be detected in different conditions.

Overall, by improving the GQD preparation conditions through the solvothermal route, the fluorescence quantum yield was enhanced. GQDs produced in this study exhibit sound stability, low level of cytotoxicity, and easy entrance to cells. GQDs could generate clear images of cells with remarkable imaging stability. Therefore, GQDs have potential applications in the fields of biological imaging and probes.

\section{Competing Interests}

There is no conflict of interests regarding the publication of this paper.

\section{Acknowledgments}

This research was supported by Foundation of Stomatology College, Lanzhou University: 20151204-2.

\section{References}

[1] S. Zhu, J. Zhang, S. Tang et al., "Surface chemistry routes to modulate the photoluminescence of graphene quantum dots: from fluorescence mechanism to up-conversion bioimaging applications," Advanced Functional Materials, vol. 22, no. 22, pp. 4732-4740, 2012.

[2] M. Zhang, L. Bai, W. Shang et al., "Facile synthesis of watersoluble, highly fluorescent graphene quantum dots as a robust biological label for stem cells," Journal of Materials Chemistry, vol. 22, no. 15, pp. 7461-7467, 2012.

[3] H. Razmi and R. Mohammad-Rezaei, "Graphene quantum dots as a new substrate for immobilization and direct electrochemistry of glucose oxidase: application to sensitive glucose determination," Biosensors and Bioelectronics, vol. 41, no. 1, pp. 498-504, 2013.

[4] R. Liu, D. Wu, X. Feng, and K. Müllen, "Bottom-up fabrication of photoluminescent graphene quantum dots with uniform morphology," Journal of the American Chemical Society, vol. 133, no. 39, pp. 15221-15223, 2011.

[5] X. Wang, K. Qu, B. Xu, J. Ren, and X. Qu, "Microwave assisted one-step green synthesis of cell-permeable multicolor photoluminescent carbon dots without surface passivation reagents," 
Journal of Materials Chemistry, vol. 21, no. 8, pp. 2445-2450, 2011.

[6] H. Li, X. He, Y. Liu et al., "One-step ultrasonic synthesis of water-soluble carbon nanoparticles with excellent photoluminescent properties," Carbon, vol. 49, no. 2, pp. 605-609, 2011.

[7] D. Pan, J. Zhang, Z. Li, and M. Wu, "Hydrothermal route for cutting graphene sheets into blue-luminescent graphene quantum dots," Advanced Materials, vol. 22, no. 6, pp. 734-738, 2010.

[8] Y. Li, Y. Hu, Y. Zhao et al., "An electrochemical avenue to greenluminescent graphene quantum dots as potential electronacceptors for photovoltaics," Advanced Materials, vol. 23, no. 6, pp. 776-780, 2011.

[9] S. Zhu, J. Zhang, C. Qiao et al., "Strongly green-photolumilinebreak nescent graphene quantum dots for bioimaging applications," Chemical Communications, vol. 47, no. 24, pp. 68586860, 2011.

[10] M. Bruchez Jr., M. Moronne, P. Gin, S. Weiss, and A. P. Alivisatos, "Semiconductor nanocrystals as fluorescent biological labels," Science, vol. 281, no. 5385, pp. 2013-2016, 1998.

[11] Z. Zhang, J. Hao, J. Zhang, B. Zhang, and J. Tang, "Protein as the source for synthesizing fluorescent carbon dots by a onepot hydrothermal route," RSC Advances, vol. 2, no. 23, pp. 85998601, 2012.

[12] P. Anilkumar, X. Wang, L. Cao et al., "Toward quantitatively fluorescent carbon-based "quantum" dots," Nanoscale, vol. 3, no. 5, pp. 2023-2027, 2011.

[13] B. I. Lemon and R. M. Crooks, "Preparation and characterization of dendrimer-encapsulated CdS semiconductor quantum dots," Journal of the American Chemical Society, vol. 122, no. 51, pp. 12886-12887, 2000.

[14] C. Liu, P. Zhang, X. Zhai et al., "Nano-carrier for gene delivery and bioimaging based on carbon dots with PEI-passivation enhanced fluorescence," Biomaterials, vol. 33, no. 13, pp. 36043613, 2012.

[15] J. L. Pelley, A. S. Daar, and M. A. Saner, "State of academic knowledge on toxicity and biological fate of quantum dots," Toxicological Sciences, vol. 112, no. 2, pp. 276-296, 2009.

[16] L. W. Zhang, W. Bäumer, and N. A. Monteiro-Riviere, "Cellular uptake mechanisms and toxicity of quantum dots in dendritic cells," Nanomedicine, vol. 6, no. 5, pp. 777-791, 2011.

[17] W. Zhang, K. Lin, Y. Miao et al., "Toxicity assessment of zebrafish following exposure to CdTe QDs," Journal of Hazardous Materials, vol. 213-214, pp. 413-420, 2012.

[18] C. H. Park, H. Yang, J. Lee et al., "Multicolor emitting block copolymer-integrated graphene quantum dots for colorimetric, simultaneous sensing of temperature, $\mathrm{pH}$, and metal ions," Chemistry of Materials, vol. 27, no. 15, pp. 5288-5294, 2015.

[19] L. R. Radovic and B. Bockrath, "On the chemical nature of graphene edges: origin of stability and potential for magnetism in carbon materials," Journal of the American Chemical Society, vol. 127, no. 16, pp. 5917-5927, 2005.

[20] Y.-S. Liu, Y. Sun, P. T. Vernier, C.-H. Liang, S. Y. C. Chong, and M. A. Gundersen, "pH-sensitive photoluminescence of $\mathrm{CdSe} / \mathrm{ZnSe} / \mathrm{ZnS}$ quantum dots in human ovarian cancer cells," Journal of Physical Chemistry C, vol. 111, no. 7, pp. 2872-2878, 2007.

[21] W. C. W. Chan and S. Nie, "Quantum dot bioconjugates for ultrasensitive nonisotopic detection," Science, vol. 281, no. 5385, pp. 2016-2018, 1998.
[22] L. A. Ponomarenko, F. Schedin, M. I. Katsnelson et al., "Chaotic dirac billiard in graphene quantum dots," Science, vol. 320, no. 5874, pp. 356-358, 2008.

[23] Y. W. Son, M. L. Cohen, and S. G. Louie, "Half-metallic graphene nanoribbons," Nature, vol. 444, pp. 347-349, 2006. 

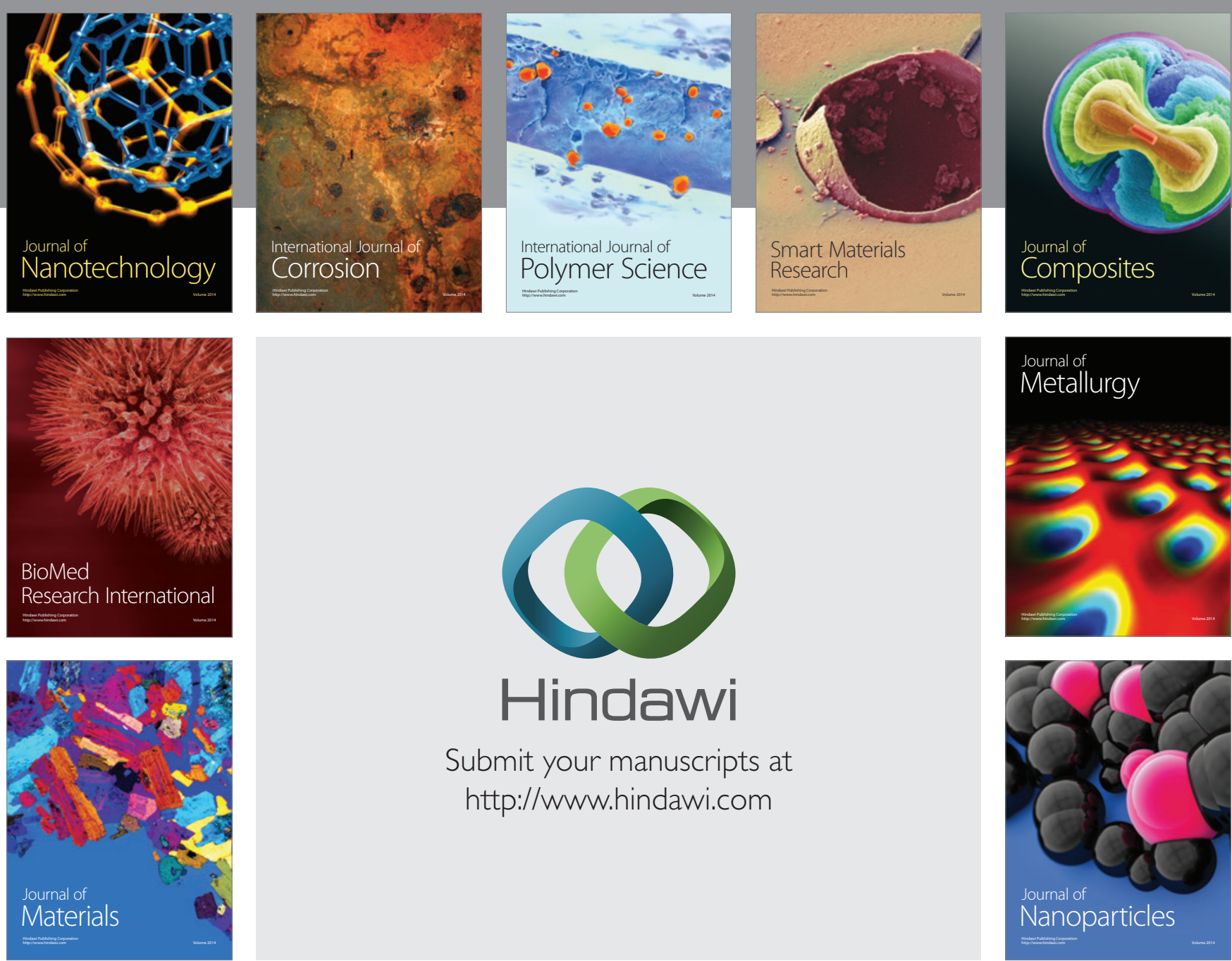

\section{Hindawi}

Submit your manuscripts at

http://www.hindawi.com

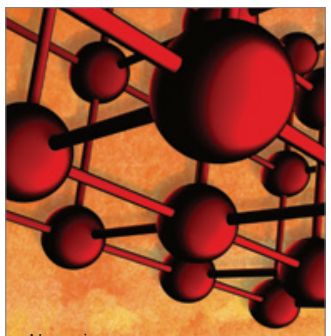

Materials Science and Engineering
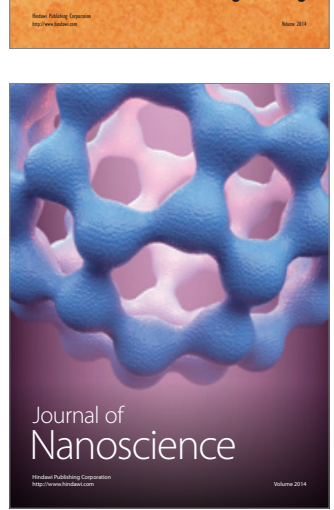
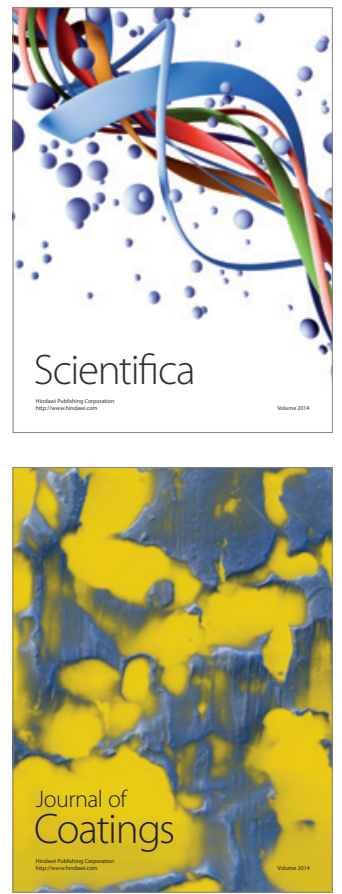
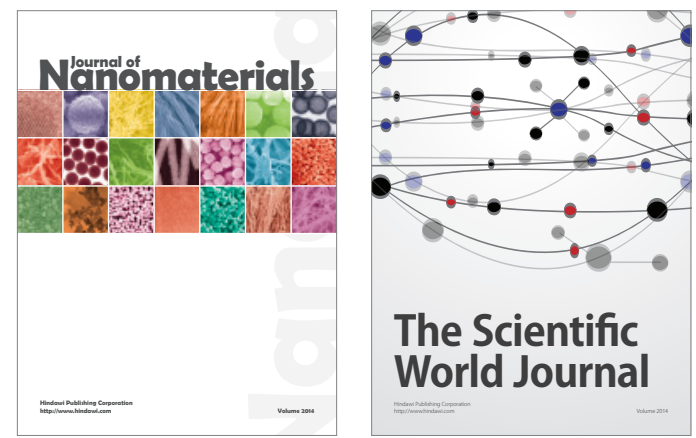

The Scientific World Journal
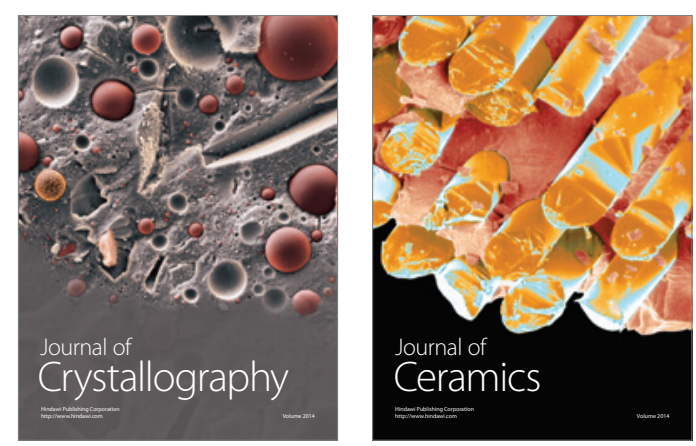
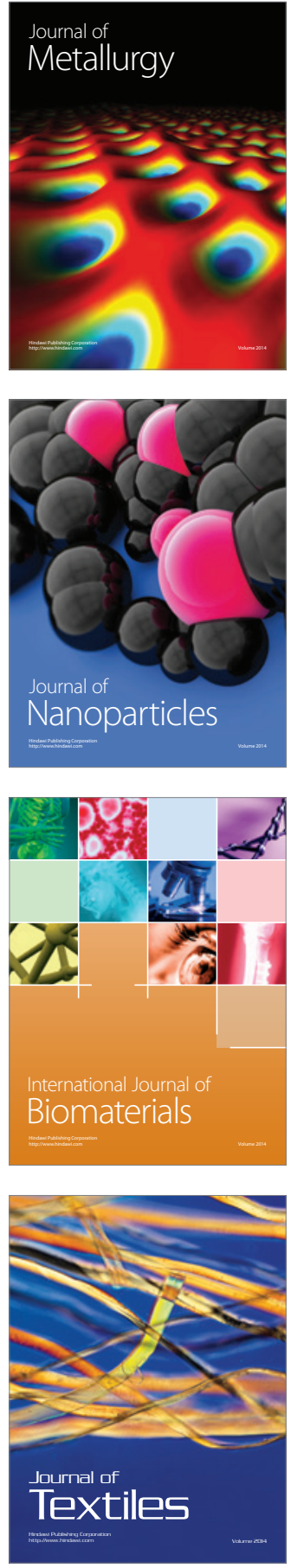